\title{
P I 3-02. Cytoskeletal proteins bound to HSP70 may elicit resistance to SIV infection of CD4+ T cells
}

\author{
L Bergmeier*1, K Babaahmady², J Pido-Lopez², K Heesom³, CG Kelly², \\ T Whittall ${ }^{2}$ and T Lehner ${ }^{2}$
}

\author{
Address: ${ }^{1}$ Centre for Clinical and Diagnostic Oral Sciences, Queen Mary, University of London, London, UK, ${ }^{2}$ King's College, London, UK and \\ ${ }^{3}$ University of Bristol, Bristol, UK \\ * Corresponding author
}

from AIDS Vaccine 2009

Paris, France. 19-22 October 2009

Published: 22 October 2009

Retrovirology 2009, 6(Suppl 3):PI84 doi:10.II86/1742-4690-6-S3-PI84

This abstract is available from: http://www.retrovirology.com/content/6/S3/PI84

(c) 2009 Bergmeier et al; licensee BioMed Central Ltd.

\section{Background}

Immunization of macaques with SIV grown in human T cells induces significant protection from infection (85\%). HSP70 isolated from these cells may carry the protective host antigens. HSP70, a potent stimulator of CC chemokine and cytokine production has an important role in innate and adaptive immune responses through its binding to CCR5, CD40 and TLR4 and has been shown to upregulate the anti HIV activity of APOBEC3G (A3G).

\section{Methods}

Two affinity purified moieties of HSP70 were extracted from human CD4+ T cells; the ADP-HSP (retaining bound proteins) or ATP-HSP (no bound proteins). Proteomic analysis of the preparations was carried out using 2D SDS-PAGE followed by MALDI-tof mass spectrometry. Two groups of macaques were immunized with the preparations (x3) at monthly intervals. SIV infectivity studies were carried out ex vivo before and after immunization. Serum antibodies were analysed by Elisa and A3G were determined by RT-PCR. The effect of cytoskeletal proteins on A3G levels in CD4+ cells were also investigated.

\section{Results}

Immunization with ADP-HSP showed significant inhibition of SIVmac251 infectivity ex vivo ( $\mathrm{p}=0.01)$, unlike ATP-HSP preparations. Proteomic analysis identified 3 cytoskeletal proteins exclusive to the ADP-HSP preparation; cofilin, profilin and gamma actin. Antibodies to cofi- lin and profilin were significantly increased post immunization in the ADP-HSP animals ( $\mathrm{p}=0.030$ and $\mathrm{p}$ $=0.026$ respectively). Antibodies to gamma actin just failed to reach significance $(p=0.078)$. There was a significant increase in A3G levels post immunization in the ADP-HSP animals $(p=0.040)$ and on stimulation of PBMC with cofilin, profilin and actin $(\mathrm{p}=0.010, \mathrm{p}=$ 0.018 and $\mathrm{p}=0.012$ respectively).

\section{Conclusion}

Investigation of the mechanism of prevention of SIV replication suggests that antibodies to the cytoskeletal proteins, may inhibit actin depolymerization and facilitate viral degradation by the innate anti-viral A3G. As cytoskeletal proteins are critical in the formation of virological and immunological synapses, finding specific antibodies and anti-SIV/HIV factors suggests a novel insight into HIV-1 immunopathogenesis. 